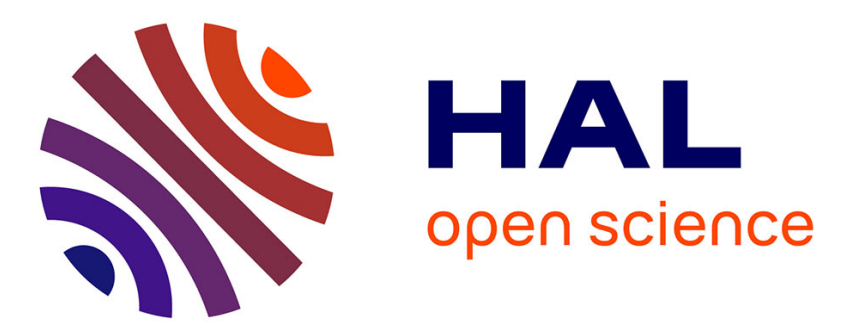

\title{
Impact of iron coordination isomerism on pyoverdine recognition by the FpvA membrane transporter of Pseudomonas aeruginosa
}

\author{
Benjamin Bouvier, Christine Cézard
}

\section{- To cite this version:}

Benjamin Bouvier, Christine Cézard. Impact of iron coordination isomerism on pyoverdine recognition by the FpvA membrane transporter of Pseudomonas aeruginosa. Physical Chemistry Chemical Physics, 2017, 19 (43), pp.29498-29507. 10.1039/c7cp04529h . hal-02395186

\section{HAL Id: hal-02395186 \\ https://hal.science/hal-02395186}

Submitted on 9 Dec 2019

HAL is a multi-disciplinary open access archive for the deposit and dissemination of scientific research documents, whether they are published or not. The documents may come from teaching and research institutions in France or abroad, or from public or private research centers.
L'archive ouverte pluridisciplinaire HAL, est destinée au dépôt et à la diffusion de documents scientifiques de niveau recherche, publiés ou non, émanant des établissements d'enseignement et de recherche français ou étrangers, des laboratoires publics ou privés. 


\title{
Impact of iron coordination isomerism on pyoverdine recognition by the FpvA membrane transporter of Pseudomonas aeruginosa ${ }^{\dagger}$
}

\author{
Benjamin Bouvier* and Christine Cézard
}

\begin{abstract}
Pyoverdines, the primary siderophores of Pseudomonas bacteria, scavenge the iron essential to bacterial life in the outside medium and transport it back into the periplasm. Despite their relative simplicity, pyoverdines feature remarkably flexible recognition characteristics whose origins at the atomistic level remain only partially understood: the ability to bind other metals than ferric iron, the capacity of outer membrane transporters to recognize and internalize noncognate pyoverdines from other pseudomonads... One of the less examined factors behind this polymorphic recognition lies in the ability for pyoverdines to bind iron with two distinct chiralities, at the cost of a conformational switch. Herein, we use free energy simulations to study how the stereochemistry of the iron chelating groups influences the structure and dynamics of two common pyoverdines and impacts their recognition by the FpvA membrane transporter of $P$. aeruginosa. We show that conformational preferences for one metal binding chirality over the other, observed in solution depending on the nature of the pyoverdine, are canceled out by the FpvA transporter, which recognizes both chiralities equally well for both pyoverdines under study. However, FpvA discriminates between pyoverdines by altering the kinetics of stereoisomer interconversion. We present structural causes of this intriguing recognition mechanism and discuss its possible significance in the context of the competitive scavenging of iron.
\end{abstract}

\section{Introduction}

Pseudomonas aeruginosa is an opportunistic Gram-negative bacterium which infects humans with weakened immune systems, leading to severe chronic and/or life-threatening conditions. Exhibiting multiple resistances to commonly used antibiotics, ${ }^{1-3}$ it has become a widespread public health issue, to the point that it has recently been placed by the World Health Organization on the list of 'superbugs' against which finding new therapeutic avenues is considered critical. ${ }^{4}$ Among these, targeting the bacterium's metal dependence has proved a promising lead. ${ }^{5}$ In particular, $P$. aeruginosa competes with its host for the acquisition of iron, which is essential to its survival and growth yet often quite scarce in the

Laboratoire de Glycochimie, des Antimicrobiens et des Agroressources, CNRS UMR7378/Université de Picardie Jules Verne, 10 rue Baudelocque, 80039 Amiens Cedex, France.

*To whom correspondence should be addressed.E-mail: benjamin.bouvier@u-picardie.fr $\dagger$ Electronic Supplementary Information (ESI) available: stereochemistry of iron chelation; additional computational details; comparison of methods for determining the chirality of octahedral complexes; statistics of dihedral angles used to define chirality; convergence of free energy calculations; structures of $\Lambda, \Delta$ and transition states of free and bound PVDI and PVD $\mathrm{G173}_{3}$; details of PCA analyses. See DOI: 10.1039/b000000x/

The calculations presented herein were performed in part using HPC resources from the MeCS computing platform of Université de Picardie Jules Verne, Amiens, France. biological medium. Under conditions of iron deprivation, the bacterium produces pyoverdine, its main siderophore, and excretes it into the outside medium where it recognizes and binds $\mathrm{Fe}^{3+}$ ions. The siderophore and its payload are then repatriated into the cell using a specific ATP-dependent transport system. ${ }^{6,7}$ Pyoverdines provide surprisingly efficient yet versatile recognition properties in a diminutive package; within their specific role, many pyoverdines show remarkable flexibility: in addition to $\mathrm{Fe}^{3+}$, they can also bind and/or transport numerous other metal cations $\left(\mathrm{Ag}^{+}, \mathrm{Al}^{3+}, \mathrm{Cd}^{2+}\right.$, $\mathrm{Co}^{2+}, \mathrm{Cr}^{2+}, \mathrm{Cu}^{2+}, \mathrm{Eu}^{3+}, \mathrm{Ga}^{3+}, \mathrm{Hg}^{2+}, \mathrm{Mn}^{2+}, \mathrm{Ni}^{2+}, \mathrm{Pb}^{2+}, \mathrm{Sn}^{2+}$, $\left.\mathrm{Tb}^{3+}, \mathrm{Tl}^{+}, \mathrm{Zn}^{2+}\right),{ }^{8,9}$ and recognize the outer membrane receptors of other Pseudomonads than the one they were synthetized by. ${ }^{10,11}$. The origin of the versatility of pyoverdine recognition, which allows bacteria to adapt to extreme conditions of iron deprivation and competitive growth, ${ }^{12}$ is to date not completely understood at the molecular level. Its mastery is essential for the design of pyoverdine analogs as noncompetitive inhibitors of bacterial membrane transporters, or Trojan horses facilitating the delivery of antibiotics, ${ }^{13}$ and made all the more urgent by the fact that new siderophore receptors are continuously being discovered. ${ }^{14}$

Pyoverdines consist of two structural domains. A fluorescent chromophore based on 2,3-diamino-6,7-dihydroquinoline, found in pyoverdines from most Pseudomonas species, binds $\mathrm{Fe}^{3+}$ via its 
catecholate function; a linear or partly cyclic polypeptide chain, specific to each species, is attached to the chromophore by its $\mathrm{N}$ terminus and chelates $\mathrm{Fe}^{3+}$ using two hydroxamate or carboxylate moieties. In initial studies, it was supposed that the conserved chromophore was mostly involved in metal binding and establishing nonspecific contacts with membrane transporters, while the variable peptide chain provided the specificity required to differenciate between bacterial strains; ${ }^{15}$ as such, the two domains were largely considered separately. Later studies revealed the interplay between both domains: on the one hand, the conformation of the peptide chain facilitates the correct positioning of the hydroxylate/hydroxamate side-chains around the metal ion; ${ }^{16}$ on the other, the coordination stereochemistry at the metal site in turn influences the folding and dynamics of the peptide chain, which play a key role in the early stages of pyoverdine recognition and binding via the establishment of transient contacts with the outer membrane transporter 'lid'. ${ }^{17}$ Based on the handedness of the helix formed by the ligands binding the metal ion, octahedral coordination complexes can have two distinct chiralities, termed $\Lambda$ and $\Delta$. Using diamagnetic $\mathrm{Ga}^{3+}$ as a probe in NMR experiments, pyoverdine-metal complexes of both chiralities have been shown to exist in solution, and sizeable differences in the conformation of their protein chains have been inferred from restraints based on NOE measurements and scalar couplings. ${ }^{18-21}$ However, the implications of this isomerism on the mechanisms of iron acquisition in Pseudomonas bacteria remain, to date, largely unknown. In particular, although the activation energy associated with the interconversion of isomers has been evaluated in the previously cited studies, the corresponding value might differ significantly in the case of the biologically relevant $\mathrm{Fe}^{3+}$ complex, for which it is not even known whether or not the two aforementioned isomers exist. Furthermore, although the order of magnitude of the activation energy hints at a transient reduction in the metal ion coordination number, ${ }^{19}$ the exact pathway for the transition between isomers is not known. Finally, the impact on the recognition of iron-laden siderophores by membrane transporters is undetermined: requiring the transporters to recognize two conformations does not make much sense, from an evolutionary point of view, if the conformational exchange has no purpose in the recognition mechanism. ${ }^{18}$
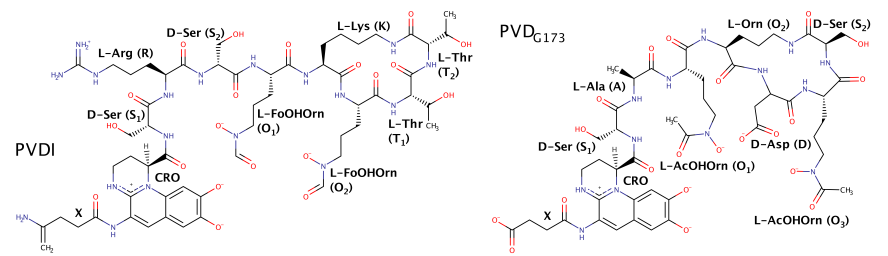

Fig. 1 Structural formulae of PVDI from Pseudomonas aeruginosa PA01 (left) and $\mathrm{PVD}_{\mathrm{G} 173}$ from Pseudomonas fluorescens G173 (right). In this paper, the aminoacids and chemical moieties are refered to using the one-letter labels between parentheses.

To shed light on these pending issues, we use long-timescale, enhanced sampling molecular dynamics to simulate the transitions between $\Lambda$ and $\Delta$ isomers for two pyoverdines: PVDI from Pseudomonas aeruginosa $\mathrm{PA} 01$, and $\mathrm{PVD}_{\mathrm{G} 173}$ from Pseudomonas fluorescens G173 (figure 1), and obtain the corresponding free energy profiles. This is done both for the siderophores in solution and for the pyoverdines bound to Pseudomonas aeruginosa's cognate TonBdependent receptor FpvA, which not only binds its native pyoverdine PVDI with very high affinity but can also recognize and bind $\mathrm{PVD}_{\mathrm{G} 173}$ (albeit with a lower affinity ${ }^{11}$ which we have evaluated computationally in previous work ${ }^{17}$ ). To our knowledge, these allatom simulations of pyoverdines bound to FpvA, embedded inside the complex lipopolysaccharide Gram-negative outer membrane, are the most realistic ever performed on these complex systems. For each pyoverdine, we discuss the relative stabilities of $\Lambda, \Delta$ and transition structures on the basis of molecular interactions. We then examine the effect of FpvA on the structure and energetics of these conformers, and discuss the differences observed between the recognition of the different forms of PVDI and $\mathrm{PVD}_{\mathrm{G} 173}$. Finally, we postulate on the role of $\Lambda-\Delta$ isomerism in the competitive scavenging of iron under conditions of deprivation.

\section{Methods}

\subsection{Stereochemistry of iron chelation}

Octahedral coordination complexes with bidentate or multidentate ligands, such as pyoverdines chelating iron, can possess chirality. The absolute configuration of such complexes is typically described based on the handedness of a helix built upon the donor atoms: complexes where the helix is left-handed are denoted by $\Lambda$, while right-handed helices give rise to $\Delta$-type chiralities. However, while the concept of helical handedness is straightforward, the actual implementation of the determination of a complex's chirality from its atomic coordinates remains awkward. Since 1961, when Piper suggested relating the handedness of a helix to a simpler relation between two skew line segments tangential to it, ${ }^{22}$ many equivalent methods have been proposed. ${ }^{23}$

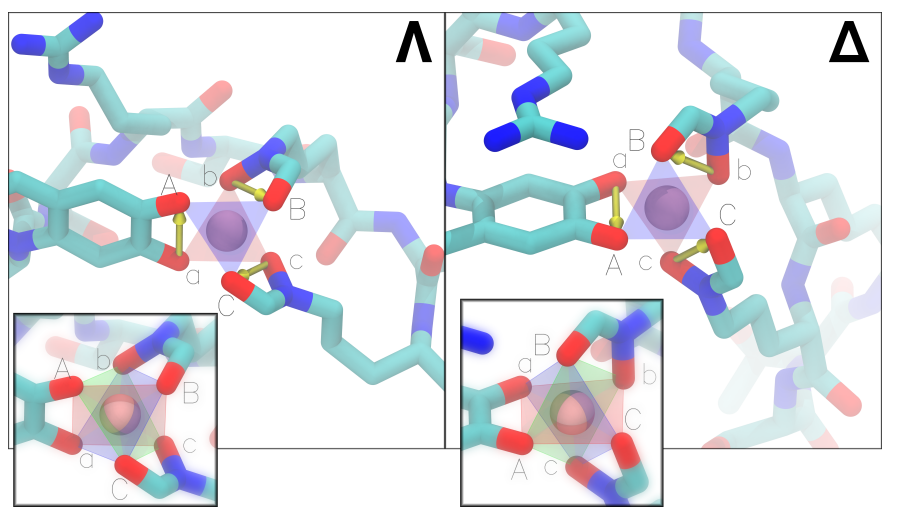

Fig. $2 \Lambda$ (left) and $\Delta$ (center) conformations of the PVDI-Fe ${ }^{3+}$ complex. The yellow arrows materialize the sense of the rotation transforming the set of chelating atoms $(\mathrm{abc})$ into $(\mathrm{ABC})$. On the insets, the three sets of four coplanar donor atoms are materialized in red, green and blue.

In this work, we define the $\Lambda$ and $\Delta$ enantiomers by the sense of the screw axis transforming the distal chelating atoms $a, b$ and c into the proximal chelating atoms A, B, and C (figure 2). The sense of the rotation can be defined by the sign of any of the dihedral angles (ACca), (CBbc) and (BAab): a negative (resp. positive) sign corresponds to a clockwise (resp. anticlockwise) rotation and defines a $\Lambda$ (resp. $\Delta$ ) isomer. Such dihedral angles are straightfor- 
ward to compute and apply biasing forces to during a simulation. For clearly defined $\Lambda$ or $\Delta$ configurations, the three angles were found to be equivalent: for practicality, we define chirality using their average, which we denote $\Phi$. In ESI, ${ }^{\dagger}$ we show the equivalence of our definition with two other alternatives to the skew line method: ${ }^{23}$ the octant sign method and the plane method. We also show that our conformational coordinate's main limitation - its ability to account for only two (one $\Lambda$ and one $\Delta$ ) out of the 16 potential stereoisomers of the $\mathrm{Fe}^{3+}$ coordination sphere $(8 \Lambda$ and $8 \Delta$ ) - does not impact the results considering that the two aforementioned isomers are compatible with NOEs and dihedral restraints from NMR experiments on PVDI binding $\mathrm{Ga}^{3+},{ }^{19}$ that the same experiments conclude that only one $\Lambda$ and one $\Delta$ isomer exist, and that no spontaneous switch to other isomers was observed during our unbiased, long-timescale molecular dynamics simulations of free PVDI or $\mathrm{PVD}_{\mathrm{G} 173}$.

\subsection{Spin state of $\mathrm{Fe}^{3+}$ bound to pyoverdines}

Iron(III) complexes can exist in different spin states depending on the distribution of the five $d$ electrons in the $3 d$ orbitals. Our $a b$ initio calculations consider all $\left[\mathrm{Fe}^{3+} \mathrm{L}_{6}\right]$ complexes as high spin, i.e. open shell systems featuring a ferric ion with five unpaired electrons $(S=5 / 2)$ and a multiplicity of six. Furthermore, spin crossover effects are neglected. Justifications for these choices, based on previous $a b$ initio studies of similar systems, can be found in ESI. ${ }^{\dagger}$

\subsection{Computational details}

Due to the relative length and complexity of the computational pipeline, only its essential features are described herein. The full computational details ensuring the reproducibility of the calculations are provided in ESI. ${ }^{\dagger}$

$A b$ initio DFT calculations. Pyoverdines are relatively large molecules in the context of $a b$ initio calculations. For such systems, DFT methods represent a good balance between accuracy and computational cost. In the last decade, many studies have shown the success and failure of different DFT methods to describe the structure, properties, energetics and reactivity of openshell $\mathrm{Fe}^{3+}$ and/or $\mathrm{Fe}^{2+}$ complexes: among the numerous functionals tested, hybrid B3LYP ${ }^{24-26}$ and $\mathrm{M06}^{27,28}$ seem to represent a sensible choice as they provide consistent results when multiple quantities have to be evaluated simultaneously. ${ }^{29,30}$ We have used these two functionals to optimize the geometry and compute the electronic energy of the two $\mathrm{Fe}^{3+}$-binding pyoverdines under study. Gibbs free energy estimates, including zero-point, thermal and entropic corrections to the electronic energies, were subsequently performed.

Molecular dynamics forcefields and parameters. All-atom simulations used the AMBER99SB forcefield ${ }^{31}$ for FpvA, the forcefield of Kirschner et $\mathrm{al}^{32}$ for LPS, the parameters of Jämbeck et al for POPE ${ }^{33}$, the TIP3P water model ${ }^{34}$, ion parameters by Joung and Cheatham, ${ }^{35}$ and pyoverdine parameters from our previous work. ${ }^{17}$ Coarse-grained simulations used the standard MARTINI force field ${ }^{36,37}$ for FpvA, POPE, water and ions, and the parameters of Van Oosten and Harroun ${ }^{38}$ for LPS. Both levels of gran- ularity employed typical simulation parameters found in state-ofthe-art simulations of corresponding systems, which are further detailed in ESI. ${ }^{\dagger}$

Molecular dynamics simulations. Setting up the simulation systems consisting of the membrane-embedded FpvA transporter in its apo state or bound to either PVDI or $\mathrm{PVD}_{\mathrm{G} 173}$ was performed in two steps: first, coarse-grained models of the systems were built and equilibrated; second, relevant coarse-grained conformations were backmapped to all-atoms and used as starting conformations for the equilibration of the corresponding all-atom systems. These two steps are summarized below.

The all-atom starting structure of the Pseudomonas aeruginosa outer membrane was taken from the work of Kirschner et $\mathrm{al}^{32}$. The LPS outer leaflet was converted to a coarse-grained representation using the grain definitions of Van Oosten et al, ${ }^{38}$ and the inner DPPE leaflet was replaced with more realistic POPE. The coarsegrained membrane was then solvated, energy-minimized and thoroughly equilibrated. Its stability (thickness, area per headgroup) was verified through $10 \mu$ s of production simulations.

The all-atom structure of the FpvA transporter was taken from the Protein Databank (id 2W75), from which the TonB-box residues were removed. It was converted to a coarse-grained representation and inserted at an optimal position into the previously equilibrated membrane patch (see $\mathrm{ESI}^{\dagger}$ for details). As before, the system was solvated, energy-minimized and equilibrated; the stability of the membrane (thickness, area per headgroup) and receptor (RMSD) were verified using $50 \mu$ s of production simulations.

A representative structure of the equilibrated system (with a small RMSD to the average structure of FpvA during the simulation) was extracted, stripped of water and chloride counterions and backmapped to all-atom. The resulting all-atom system was minimized, solvated and neutralized, and equilibrated. Production simulations of the equilibrated all-atom system were then performed for $75 \mathrm{~ns}$.

The all-atom starting structures for the simulations of PVDI and $\mathrm{PVD}_{\mathrm{G} 173}$ bound to FpvA were generated by fitting the rigid FpvA barrel $\mathrm{C}_{\alpha}$ atoms in PDB structures 2W16 (FpvA/PVDI) and 2W6U (FpvA/PVD $\mathrm{G}_{\mathrm{G} 13}$ ) to the equilibrated apo-FpvA structure obtained as described in the previous paragraphs. Equilibration simulations proceeded as for apo-FpvA. Production simulations amounted to 75 ns for each of the two systems, during which no spontaneous transition from $\Lambda$ to $\Delta$ was observed. The $\Delta$ pyoverdines bound to FpvA were simulated for the same duration, starting from structures representative of the $\Delta$ siderophores extracted from the $\Lambda / \Delta$ metadynamics simulations; similarly, no spontaneous transition to $\Lambda$ chiralities were observed.

The starting structures for the isolated pyoverdines were extracted from PDB entries 2W16 (FpvA/PVDI) and 2W6U $\left(\mathrm{FpvA} / \mathrm{PVD}_{\mathrm{G} 173}\right)$ and minimized, solvated and equilibrated as previously described. Both pyoverdines were simulated for $100 \mathrm{~ns}$, during which no conversion from $\Lambda$ to $\Delta$ was observed. Similar simulations starting from $\Delta$-type chiralities showed no flip to $\Lambda$ geometries in the same timescale.

The transitions of the isolated and FpvA-bound pyoverdines between the $\Lambda$ and $\Delta$ isomers were accelerated using well-tempered metadynamics simulations. ${ }^{39}$ The biased coordinate was the mean 
of the three dihedral angles defining the proximal and distal planes of donor atoms (see Methods and figure 2). The total simulation time was $500 \mathrm{~ns}$ for the isolated pyoverdines and $250 \mathrm{~ns}$ for the FpvA-bound siderophores. COnvergence of the free energy profiles was verified (see $\mathrm{ESI}^{\dagger}$ figure S4).

\section{Results}

\subsection{Stability and iron coordination of pyoverdine stereoiso-} mers

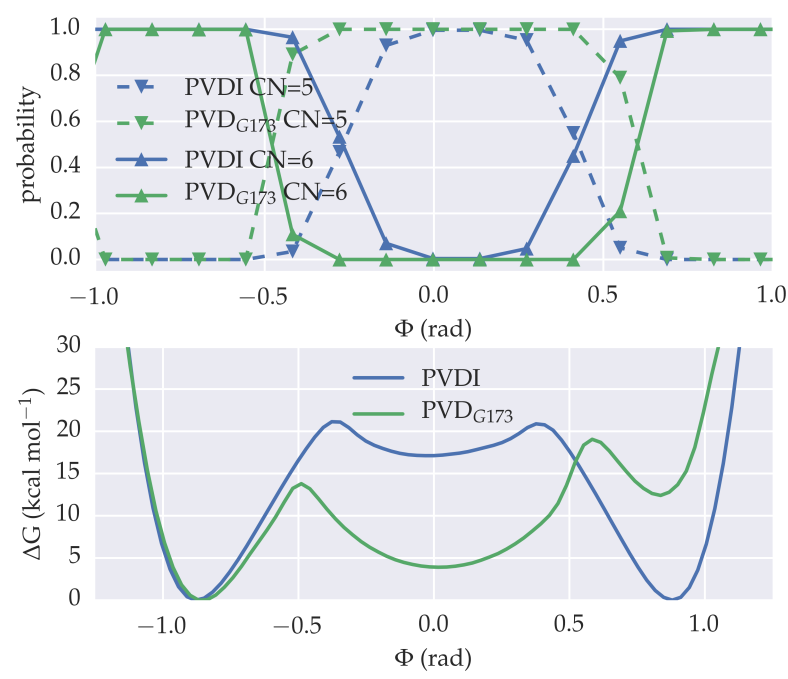

Fig. 3 Coordination and relative free energies of $\mathrm{Fe}^{3+}$-binding PVDI and $P V D_{\mathrm{G} 173}$ as a function of $\Phi$. Top: probability of encountering pentacoordinated $(\mathrm{CN}=5)$ and hexacoordinated $(\mathrm{CN}=6)$ complexes. Bottom: free energy profiles.

To unravel the mechanisms and energetics behind the $\Lambda-\Delta$ transition in pyoverdines, we performed well-tempered metadynamics simulations in which the motion of the system along the conformational coordinate $\Phi$ (see Methods) used to define chirality was amplified. This yielded the free energy profile along this coordinate for each of the pyoverdines. In addition, the coordination number of the $\mathrm{Fe}^{3+}$ ion (based on a maximum distance of $2.5 \AA$ between $\mathrm{Fe}^{3+}$ and a coordinated donor atom) was monitored along the $\Lambda^{-}$ $\Delta$ transition pathway; both plots appear on figure 3 . As can be seen, while $\Lambda$ and $\Delta$ structures are strictly hexadentate, intermediate conformations are mostly pentacoordinated, with transition regions showing mixtures of both. This applies to both pyoverdines, although the onset of pentacoordinated complexes appears slightly earlier for $\mathrm{PVD}_{\mathrm{G} 173}$ than for PVDI, in both directions $(\Lambda$ to $\Delta$ and $\Delta$ to $\Lambda$ ). The free energy profiles reflect this unfavorable loss of coordination by a barrier leading to an intermediate, metastable state; surprisingly, this state is much higher in energy for PVDI (17.1 kcal mol$\left.{ }^{-1}\right)$ than for PVD $\mathrm{G}_{173}\left(3.9 \mathrm{kcal} \mathrm{mol}^{-1}\right)$ despite the pentavalent nature of both intermediates. The difference in free energy between $\Lambda$ and $\Delta$ conformers is also strikingly different for both siderophores: while both states are isoenergetic in PVDI, $\Delta$ enantiomers of $\mathrm{PVD}_{\mathrm{G} 173}$ are $12.4 \mathrm{kcal} \mathrm{mol}^{-1}$ less stable than their $\Lambda$ counterparts.
Table 1 Enthalpy and free energy differences $\left(\mathrm{kcal} \mathrm{mol}^{-1}\right)$ between the $\Lambda$ and $\Delta$ enantiomers of PVDI and PVD $\mathrm{G}_{173}$ calculated at the DFT level of theory with two different functionals.

\begin{tabular}{lrrrr}
\hline & \multicolumn{2}{c}{ B3LYP } & \multicolumn{2}{c}{ M06 } \\
\cline { 2 - 5 } & $\Delta \mathrm{H}$ & $\Delta \mathrm{G}$ & $\Delta \mathrm{H}$ & $\Delta \mathrm{G}$ \\
\hline PVDI & -6.60 & -4.46 & -4.80 & -2.31 \\
PVD $_{G 173}$ & -23.48 & -18.47 & -22.10 & -20.52 \\
\hline
\end{tabular}

Although our pyoverdine force field parameters have previously been shown to faithfully reproduce experimental free energies, ${ }^{17}$, the very crude treatment of transition metal ions such as $\mathrm{Fe}^{3+}$ inside a classical framework could cast doubt upon the reported free energy profiles. As a validation, we performed density functional calculations, using two different functionals, on the reoptimized structures of the $\Lambda$ and $\Delta$ enantiomers of each pyoverdine obtained from our simulations (Table 1 ). The free energy differences based on DFT calculations cannot be directly compared with those extracted from MD simulations, because of the very basic treatment of configurational entropy in the former and the coarse description of molecular interactions in the latter. However, it is quite clear that, at both levels of theory, there is a very large free energy gap between the $\Lambda$ and $\Delta$ conformers of $\mathrm{PVD}_{\mathrm{G} 173}$ (in favor of the former) whereas for PVDI both can be considered to be roughly isoenergetic.

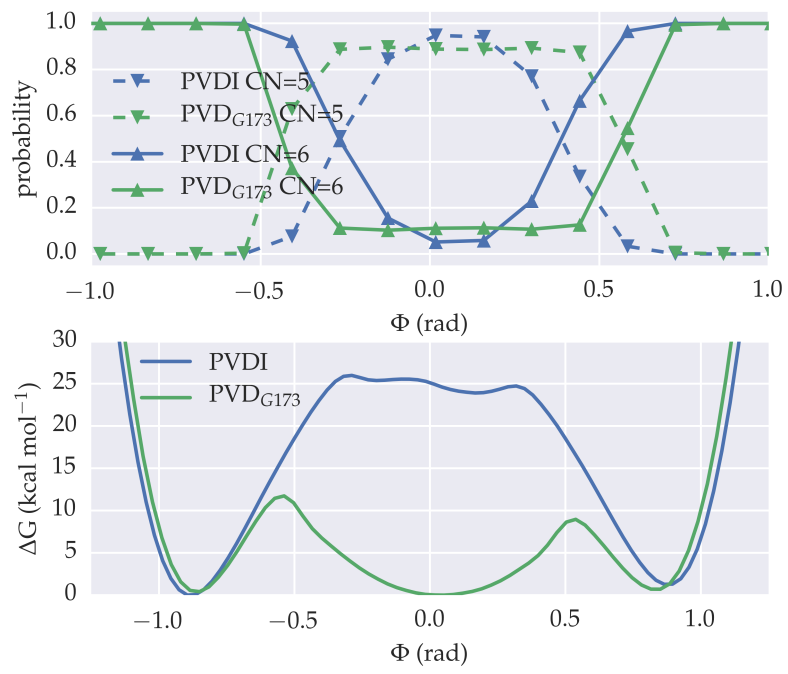

Fig. 4 Coordination and relative free energies of $\mathrm{Fe}^{3+}$-laden PVDI and $\mathrm{PVD}_{\mathrm{G} 173}$ bound to FpvA as a function of $\Phi$. Top: probability of encountering pentacoordinated $(\mathrm{CN}=5)$ and hexacoordinated $(\mathrm{CN}=6)$ complexes. Bottom: free energy profiles.

We now compare the free energies and coordinations along the $\Lambda-\Delta$ transition for the pyoverdines bound to FpvA (figure 4). The interaction with FpvA has a relatively minute effect for PVDI: the transition barrier is slightly raised and the intermediate state, slightly destabilized, no longer corresponds to a local free energy minimum. The effect on $\mathrm{PVD}_{\mathrm{G} 173}$ is much more striking, with an impressive stabilization of both intermediate states and $\Delta$ enantiomers down to the level of the $\Lambda$ structures. 
A possible limitation of our simulation methodology stems from the fact that the $\Delta$ conformations of the siderophores originate from simulations whose starting points are crystal structures of $\Lambda$ chiralities, which are the only ones available. This could raise an issue for the FpvA-bound pyoverdines if the binding modes of $\Delta$ and $\Lambda$ conformers to the transporter were very different and separated by a large free energy barrier: the bias and timescales of the metadynamics simulations might not offer the siderophore the opportunity to unbind and rebind upon switching chiralities. However, considering the predicted relative stability of the $\Delta$ states and the fact that the overall binding modes are mostly imposed by nonspecific contacts with the chromophore, ${ }^{11}$ we do not expect the existence of alternate $\Delta$ siderophore binding modes very likely. Furthermore, for the $\Lambda$ state of PVDI, there is experimental evidence that the bound and free conformations share the same metal coordination sphere; ${ }^{19}$ this probably also holds true for bound and free $\Delta$ conformers (see $\mathrm{ESI}^{\dagger}$ for details).

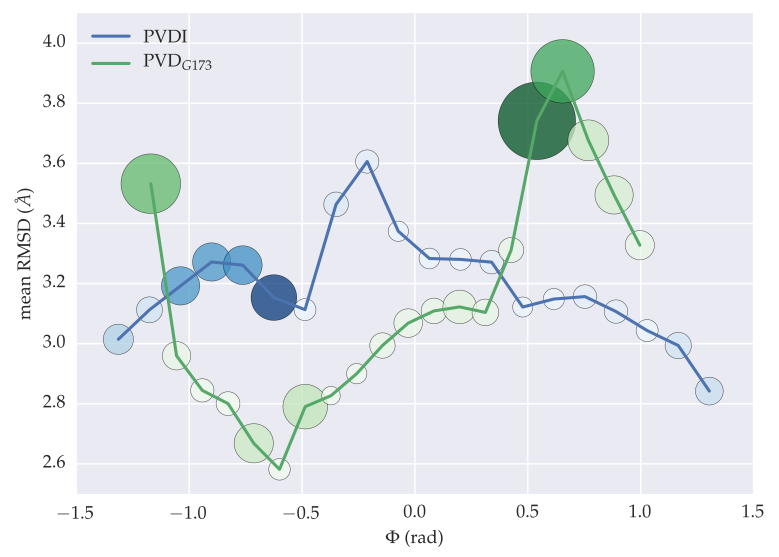

Fig. 5 Evolution of the average RMSD ( $y$ axis) and Hausdorff distance (marker circle diameter) between the isolated and FpvA-bound pyoverdines as a function of $\Phi$.

3.2 The conformational landscapes of pyoverdines are defined by rigid and flexible parts bridged by the third chromophore ring

Pyoverdines are intricate molecules which span the gap between small ligands and proteins; their conformational space is likely accordingly complex. To discover the reasons behind the differences in free energy profiles and their impact on stereospecific siderophore recognition, we focus on the conformational differences between bound and unbound pyoverdines along the $\Lambda-\Delta$ pathway. To achieve this, we divide the pathway into 20 bins of equal width along $\Phi$, into which we distribute the conformations from our simulations. We then compute the RMSD between all possible free-bound pairs of conformations falling within the same bin. On figure 5, we represent two statistics over these RMSD sets: (i) the mean RMSD value gives an idea of the typical structural divergence between the most frequently occurring bound and unbound structures; (ii) the Hausdorff distance quantifies the overlap between conformational subsets: clearly separated (resp. overlap- ping) subsets are associated with large (resp. small) Hausdorff distances.

The evolution of both the average and Hausdorff distances between the RMSD sets of free and bound PVDI can be seen to be rather contained throughout the pathway. Interestingly, the peak in Hausdorff distances does not coincide with the peak in average RMSD, indicating that it is associated with an unfavorable reduction of conformational entropy in the barrier area rather than the separation of free and bound pathways. Similarly, the peak in average RMSD being associated with a small Hausdorff distance hints at a recentering of the free and bound conformational densities without major changes in the overall spanned conformational space, rather than a movement of the bound and unbound subsets away from each other. We conclude that the isomerization mechanism in the free and bound PVDI can be said to be largely similar. Looking at the structures (ESI ${ }^{\dagger}$ figure S5) corroborates these assumptions: the free and bound $\Lambda$ and $\Delta$ states are remarkably close, essentially differing by the orientation of the chromophore substituent group and arginine side chain. As previously discussed, the intermediate state chelates $\mathrm{Fe}^{3+}$ pentavalently in both cases: the four chelating oxygen atoms of the chromophore and the second ornithine residue $\left(\mathrm{O}_{2}\right)$ form a plane also containing $\mathrm{Fe}^{3+}$; meanwhile, the first ornithine $\left(\mathrm{O}_{1}\right)$ retains its connection to the metal ion via the nitroso oxygen only. However, the conformation of the lysine side chain is different in the free and bound states, resulting in different positionings of the cyclic peptide moiety (and the hydrogen-bond donor threonine residues contained therein).

In the case of $\mathrm{PVD}_{\mathrm{G} 173}$, both average RMSD and Hausdorff distances peak at the onset of the $\Delta$ conformer, rising sharply in the barrier region separating the latter from the intermediate state; conversely, the free and bound $\Lambda$ basins are rather similar according to both measures. This indicates a clear divergence of the free and bound conformational pathways when moving away from the intermediate state and toward $\Delta$. Representative free and bound $\mathrm{PVD}_{\mathrm{G} 173}$ structures (ESI ${ }^{\dagger}$ figure S6) confirm this picture: while $\Lambda$ and intermediate states appear mostly comparable, distinct puckerings of the chromophore third ring induce clear conformational divergences in the peptide backbone of the free and bound $\Delta$ states.

This reveals the pucker state of the third chromophore ring as a good indicator of the possible divergence of pathways between bound and free siderophores (figure 6). PVDI exists in a balanced mixture of C1-exo and N4-endo conformations for relevant values of $\Phi$, regardless of whether the siderophore is bound to FpvA. On the other hand, the preference for C1-endo/C2-exo conformations in the free and bound $\Lambda$ enantiomer of $\mathrm{PVD}_{\mathrm{G} 173}$ rapidly evolves toward C1-exo in the bound state, while for the free siderophore this transition happens much later along the $\Lambda-\Delta$ pathway. This switch in pucker states in $\mathrm{PVD}_{\mathrm{G} 173}$ marks the onset of the conversion from $\Lambda$ to $\Delta$, triggering conformational changes in the proximal peptide residues which, in turn, profoundly alter the position of the distal cyclic oligopeptide chain. However, these conformational changes propagate slowly along the pathway: while different pucker states are already visible for the intermediate state (see ESI ${ }^{\dagger}$ figure S6), their impact on the peptide backbone only becomes apparent inside the $\Delta$ basin.

Another striking difference between the isomerization pathways 


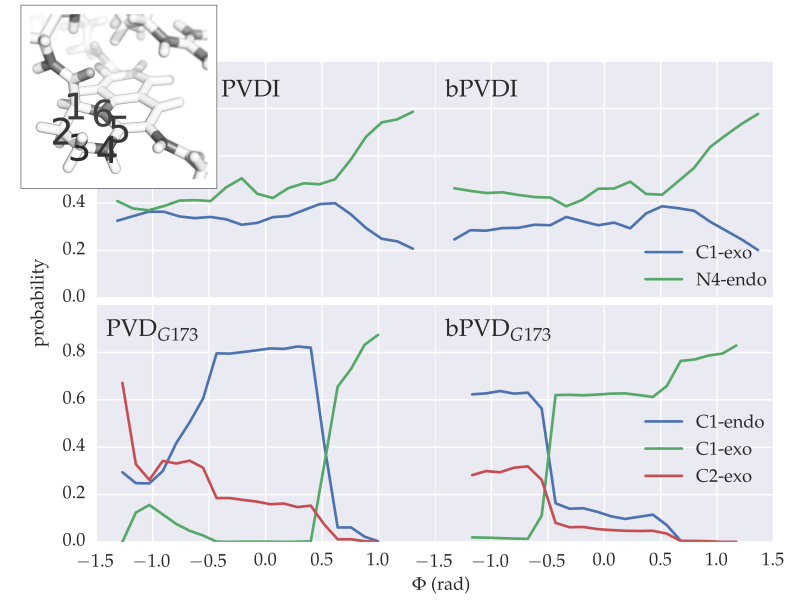

Fig. 6 Dominant pucker states of the third chromophore ring (inset) for the free and bound (b prefix) pyoverdines as a function of $\Phi$.

of PVDI and $\mathrm{PVD}_{\mathrm{G} 173}$ concerns the distribution of chelating atoms inside the metal coordination sphere for intermediate states: while both intermediate states feature pentacoordinated $\mathrm{Fe}^{3+}$ ions, the partially decoupled hydroxamate function belongs to the first ornithine $\left(\mathrm{O}_{1}\right)$ in PVDI and to the second $\left(\mathrm{O}_{3}\right)$ in $\mathrm{PVD}_{\mathrm{G} 173}$. In $\mathrm{PVD}_{\mathrm{G} 173}, \mathrm{O}_{3}$ is positioned on the other side of the chromophore plane, opposite the main peptide chain, resulting in a compact structure in which the metal ion is thoroughly shielded; meanwhile, in PVDI the partially unbound $\mathrm{O}_{1}$ is on the same side as the peptide chain, inducing a more elongated conformation with a much more accessible $\mathrm{Fe}^{3+}$.

\subsection{The subtle competition between intra- and intermolecu- lar interactions powers chiral recognition}

The free energy and conformational divergences descibed above are linked to a competition between self-interactions within the pyoverdines and interactions with water and/or the FpvA transporter. We focus now on how these interactions are used to leverage the chiral recognition of siderophores.

The first aspect concerns the role of water in the stabilization of the pentacoordinated intermediate states. Figure 7 presents the radial distribution functions ( $\mathrm{RDF}$ ) of water around $\mathrm{Fe}^{3+}$ for the relevant chiralities of both free pyoverdines. As can be seen, the first hydration layer in the $\Lambda$ and $\Delta$ states is rather remote from the ion (with the corresponding peak centered at $3.3 \AA$ in both cases). This layer appears denser and more structured for PVDI than for $\mathrm{PVD}_{\mathrm{G} 173}$, for which the density is somewhat shifted to the third peak and beyond; this is likely due to the overall more hydrophobic character of the $\mathrm{PVD}_{\mathrm{G} 173}$ peptide chain. Apart from a slightly more structured second solvation layer for $\Delta \mathrm{PVD}_{\mathrm{G} 173}$, the chirality of the siderophores has little impact on the solvation patterns. Conversely, the reduced coordination of $\mathrm{Fe}^{3+}$ in the intermediate states opens up space for water molecules to come into direct contact with the ion, resulting in the apparition of two additional, highly structured water layers. These water layers are both closer and more structured for $\mathrm{PVD}_{\mathrm{G} 173}$ than for PVDI, for reasons which become apparent when looking at the structures.
In both cases, a water molecule restores an octahedral layout of donor atoms around the metal by interacting with $\mathrm{Fe}^{3+}$ on the opposite side of the partially unbound ornithine. In $\mathrm{PVD}_{\mathrm{G} 173}$, this molecule is involved in a hydrogen bond with the peptide backbone which locks it into place relative to the siderophore. In PVDI, the water molecule cannot contact the peptide backbone which lies on the other side of the chromophore plane; it can only do so indirectly via a chain of hydrogen-bonded water molecules, which tends to rigidify the third solvation layer. In agreement with the free energy profiles, the stabilization of the pentavalent intermediate is thus more favorable in $\mathrm{PVD}_{\mathrm{G} 173}$, both from the enthalpic and entropic points of view. A similar trend can be observed for the RDFs of the bound pyoverdines (data not shown), proving that the pyoverdine binding site of FpvA is spacious enough not to hinder the arrangement of the $\mathrm{Fe}^{3+}$ hydration shell.

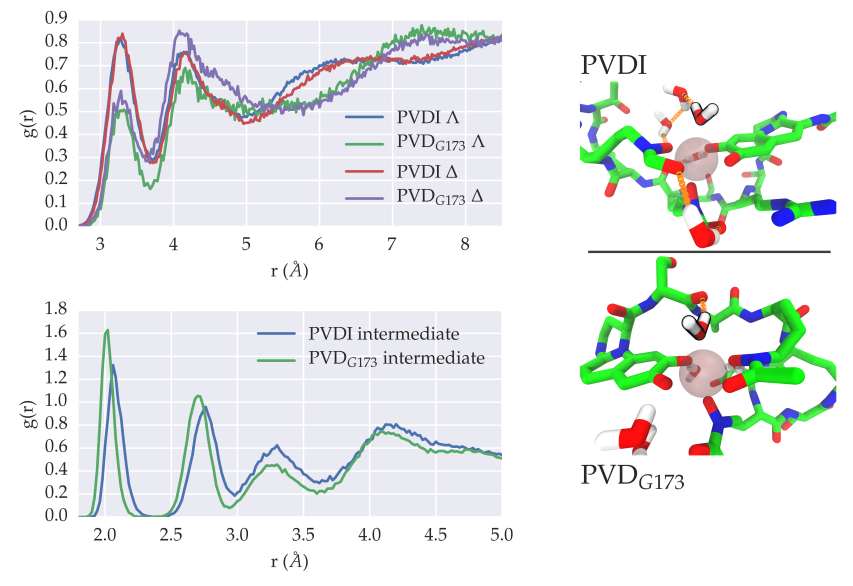

Fig. $7 \mathrm{RDF}$ of water around $\mathrm{Fe}^{3+}$ for $\Lambda, \Delta$ (top left) and intermediate (bottom left) states of PVDI and PVD $\mathrm{G}_{173}$. Right: representative structures of the first water layers around $\mathrm{Fe}^{3+}$ for the intermediate states of PVDI (top) and PVD $\mathrm{G}_{173}$ (bottom), with hydrogen bonds as orange dashed cylinders.

The numerous hydrogen-bonding groups on the pyoverdine peptide chains can give rise to a competition between intermolecular pyoverdine-FpvA and intramolecular interactions. We examine the evolution of this competition, which probably plays a dominant role in the chiral discrimination of the siderophores, along the isomerization pathways (figure 8)

In the free PVDI, a transient intramolecular salt bridge with the arginine side chain stabilizes the dangling hydroxamate oxygen of $\mathrm{O}_{1}$ in the intermediate state; in the bound PVDI, the arginine is locked in an interaction with Y231 of FpvA and unable to play this role. This explains the higher conversion barrier and absence of stable intermediate state for FpvA-bound compared to free PVDI.

In the free $\mathrm{PVD}_{\mathrm{G} 173}$, the unbound hydroxamate is located opposite the main peptide chain and cannot be stabilized by a direct intramolecular interaction; instead, the planar disposition of four out of the five remaining oxygens chelating $\mathrm{Fe}^{3+}$ is stabilized by an intra-residue hydrogen bond between the backbone and side chain of $\mathrm{O}_{1}$. In the bound complex, the dangling ornithine $\left(\mathrm{O}_{3}\right)$ is directly stabilized by an intramolecular $\mathrm{O}_{3}-\mathrm{O}_{1}$ hydrogen bond reinforced by an $\mathrm{R} 204-\mathrm{O}_{3}$ interaction $\mathrm{CO}_{3}$ thus acts both as a donor and 
an acceptor). These interactions, complemented by a salt bridge between $\mathrm{S}_{1}$ of $\mathrm{PVD}_{\mathrm{G} 173}$ and $\mathrm{D} 597$ of the receptor, appearing in the intermediate state and growing stronger in the $\Delta$ state, explain the remarkable stabilization of these conformations of the bound $\mathrm{PVD}_{\mathrm{G} 173}$. These results confirm the importance of the FpvA plus domain bearing residues R204 and Y231; its role is obvioulsy not limited to the nonspecific recognition of the chromophore, but encompasses the specific recognition of chirality-iunduced conformations of the peptide chain. Similarly, the so-called L7 loop bearing D597, previously shown to undergo conformational changes depending on the pyoverdine strain being recognized, ${ }^{11}$ seems to have more roles than anticipated.

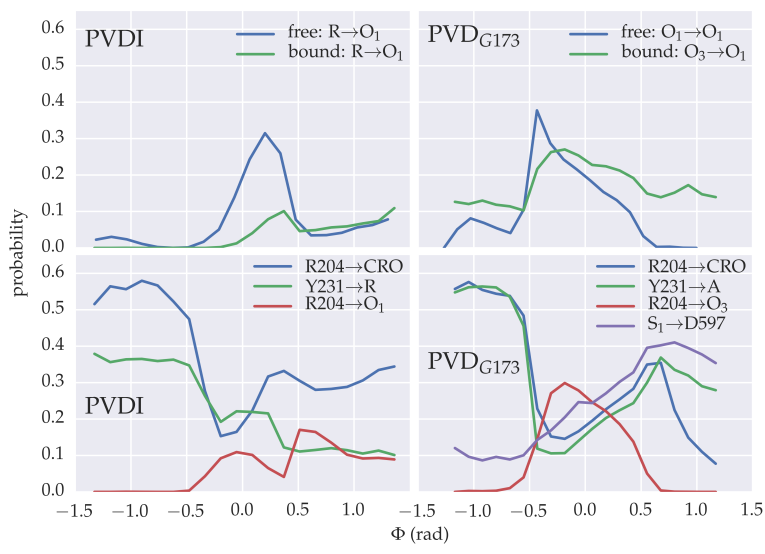

Fig. 8 Probability of occurrence of (i) the most frequent intramolecular hydrogen bonds in free and FpvA-bound PVDI (upper left) and $\mathrm{PVD}_{\mathrm{G} 173}$ (upper right); (ii) the most frequent intermolecular hydrogen bonds between FpvA and PVDI (lower left) or PVD $\mathrm{G}_{173}$ (lower right), as a function of $\Phi$. The arrows materialize the directionality of the hydrogen bonds (donor to acceptor).

Finally, we examine the influence of the siderophore stereochemistry on the dynamics of the flexible loops which form the lid of the FpvA transporter (figure 9, left panel). We applied principal component analysis (PCA) to the backbone of the loop segments to extract the correlated motion of the corresponding atoms along the $\Lambda-\Delta$ isomerization pathway, and compared with the same analysis performed on the apo transporter. On $\mathrm{ESI}^{\dagger}$ figure S7, the amplitude of the contribution of each loop to the dominant PCA eigenmodes (directly related to the amplitude of the loop motion) are represented. As expected, interactions between the FpvA lid loops and pyoverdines tend to result in a rigidification of the former upon binding of the latter. This is the case for the M431A441, W491-S519 and D699-Q710 loops, which show a reduction of flexibility regardless of the nature and chirality of the bound pyoverdine. Loops P595-D611 and E646-K664, on the other hand, appear to exhibit a more specific behavior; in particular, the $\Delta$ stereoisomer of $\mathrm{PVD}_{\mathrm{G} 173}$ triggers a marked rigidification of these loops whereas other pyoverdines retain (or even slightly enhance) the plasticity observed in the apo transporter. This is all the more surprising since E646-K664 does not make direct contact with the bound siderophores.

Interestingly, loop P595-D611 is located just below loop E646-
K664 on the pore barrel; during an outward motion, the base of E646-K664 will tend to 'push' on P595-D611. Consequently, P595D611 can potentially curb the motion of E646-K664. In the absence of strong, lasting interactions between P595-D611 and PVDI or $\Lambda \mathrm{PVD}_{\mathrm{G} 173}$, the loop is free and does not restrict the mobility of E646-K664, which is characterized by an extensive swinging motion around its base with little deformation of the loop itself (figure 9, center). For $\Delta \mathrm{PVD}_{\mathrm{G} 173}$, favorable interactions between $\mathrm{S}_{1}$ of $\Delta$ PVDG and D597 (mentioned previously) restrict the motion of loop P595-D611, in turn preventing the outward motion of E646-K664. Instead, E646-K664 adopts a completely new motion in which the loop deforms along the plane of the membrane (figure 9, right; see ESI figure S7 for details). Loop P595-D611 (often dubbed loop L7) thus acts as a switch, allowing the allosteric transfer of information between the pyoverdine binding site and the pore lid loops and amplifying the change of flexibility upon siderophore binding. Beyond the straightforward entropic contribution to the stabilization of specific conformers and structures, this mechanism is likely to play a role in the pyoverdine-mediated signaling linking both sides of the outer membrane 40 but could also link this pathway to other facets of iron metabolism, such as quorum sensing.

\section{Discussion}

Several significant findings have emerged from our simulations of the $\Lambda-\Delta$ isomerization mechanisms in PVDI and $\mathrm{PVD}_{\mathrm{G} 173}$. We summarize them here in the light of previously acquired knowledge and pending questions on the complex iron acquisition pathways in Gram-negative bacteria.

The first finding is the confirmation of the experimentally postulated coordination of the intermediate state. Based on the observation of exchange kinetics via NMR chemical shifts in $\mathrm{Ga}^{3+}$ binding PVDI, Wasielewski et $\mathrm{al}^{19}$ postulated that this state involved the breakage of one oxygen-metal bond. Our study suggests that this is also the case for the biologically relevant, $\mathrm{Fe}^{3+}$. binding siderophores. The free energy penalty involved very likely depends on the nature of the metal ion, ${ }^{18}$ making comparisons to experiments using diamagnetic $\mathrm{Ga}^{3+}$ as a substitute for paramagnetic $\mathrm{Fe}^{3+}$ somewhat qualitative despite the very similar radius of both ions. Keeping this in mind, the experimental $\Lambda$ to $\Delta$ activation enthalpy in $\mathrm{Ga}^{3+}$-binding PVDI $\left(20 \mathrm{kcal} \mathrm{mol}^{-1}\right)$ seems in line with our results. The predicted energetic degeneracy of $\Lambda$ and $\Delta$ enantiomers also matches experimental findings. These results validate the employed methodologies despite the complexity of the systems involved (transition metal ion inside a classical framework, complex Gram-negative outer membrane, combination of rigid and flexible domains inside FpvA, intricate coupling of motion on different timescales, etc). Similarly, the simple order parameter employed in our simulations successfully transforms the experimentally observed $\Lambda$ coordination sphere isomer into a $\Delta$ isomer compatible with experimental observations, showing that the most simple and direct pathway is the one actually taking place.

Considering these safeguards (further reinforced by ab initio calculations), there is little doubt that the very different relative stabilities of the intermediate and $\Delta$ states with respect to $\Lambda$ in both pyoverdines are not artefactual. The intermediate states of PVDI 


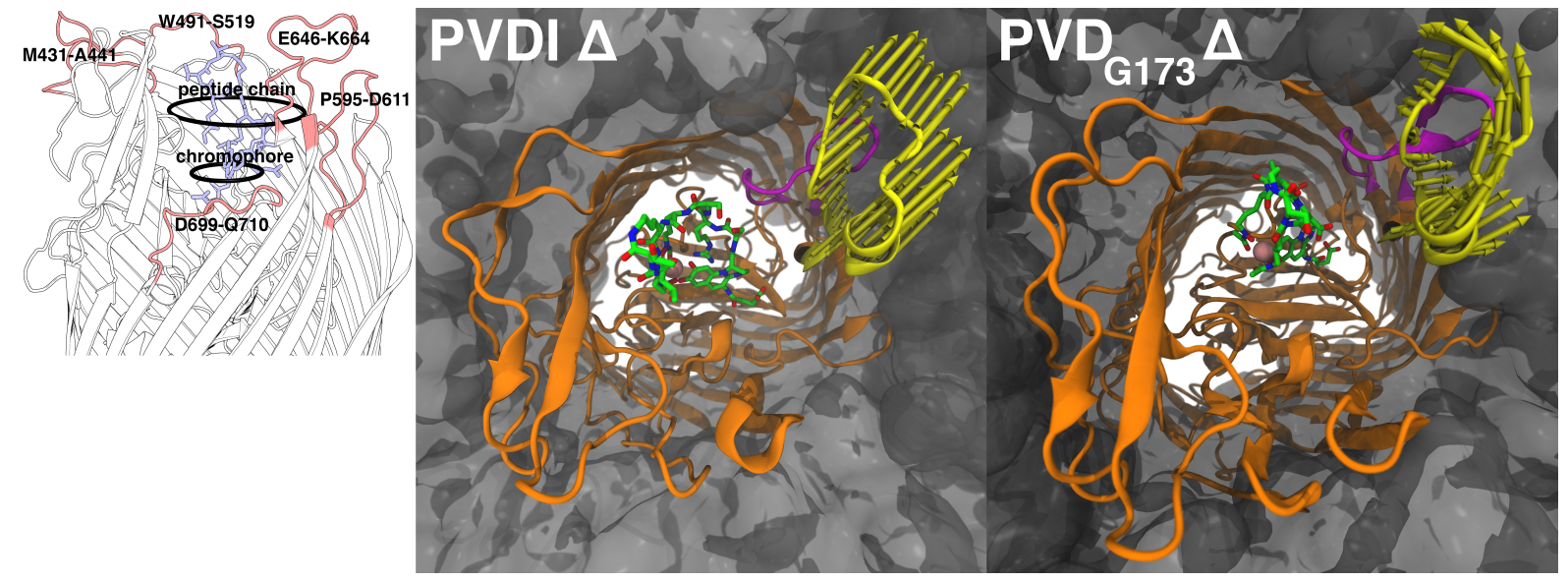

Fig. 9 Left: cartoon representation of FpvA binding PVDI, viewed along the membrane plane; red: lid loops with corresponding residue spans; blue: bound pyoverdine, with chromophore and peptide chain regions identified. Center/right: Cartoon representations of FpvA binding $\triangle$ PVDI or PVD ${ }_{G 173}$, viewed from the outside medium along the pore axis, with lid loops P595-D611 (violet) and E646-K664 (yellow) identified; the arrows represent the directionality of atomic motion of loop E646-K664 along the first PCA eigenmode.

and $\mathrm{PVD}_{\mathrm{G} 173}$ share pentavalent coordinations but differ in which of the oxygen-metal bonds has been ruptured. As a result, the compact, radial arrangement of the polypeptide chain around the metal ion in $\mathrm{PVD}_{\mathrm{G} 173}$ is quite distinct from the more elongated PVDI intermediate in which all atoms are on one side of the chromophore plane. It is intriguing to see that the pyoverdine with the shortest peptide chain adopts a more tightly folded conformation around $\mathrm{Fe}^{3+}$ than PVDI, whose longer chain would in theory have supported such a conformation with less strain. Our structures reveal that the necessity to accomodate the bulky arginine side chain of PVDI hinders a tight fit around the metal ion, which is not the case for $\mathrm{PVD}_{\mathrm{G} 173}$ and its small, equivalently positioned alanine. The NMR chemical shifts of the side-chain nuclei of arginine suggest the position of this aminoacid to be quite constrained, and likely involved in intramolecular contacts; ${ }^{18}$ our simulations show that this is indeed the case. More generally, our results highight the importance of intramolecular interactions, which often have more influence on the conformational preferences of the pyoverdines than receptor-siderophore contacts. This is the case for intermediate states: contacts with FpvA modify their relative stability and cause slightly different positionings of the distal protein chains compared to their free counterparts, but do not affect the metal coordination sphere. Pyoverdines thus implement the stringent conformational specificity of small ligands through strong intramolecular interactions, whilst retaining a limited plasticity compatible with labile intermolecular contacts, as seen in proteins; the cyclic nature of the protein chain and the semiflexible third chromophore ring mitigate these two aspects. This is likely a key point in explaining the polymorphism of pyoverdine recognition by TonB-dependent transporters.

Consequently, when designing novel antibiotics upon the pyoverdine scaffold to meet WHO recommendations, it appears worthwhile to ensure that intramolecular interactions can stabilize all possible metal-binding chiralities, to complement the relative chiral agnosticism of FpvA (discussed below) implemented by intermolecular interactions. Subtle variations in pyoverdine se- quences have already been shown by circular dichroism spectra to profoundly impact the relative stability of stereoisomers, affecting their recognition; ${ }^{11}$ our study provides the groundwork for the preliminary in silico screening of potential siderophore analogs for therapeutic purposes.

Experiments have shown that many bacteria are specific for certain siderophore chiralities. For instance, $\Lambda$ enterobactin does not promote bacterial growth, while $\Delta$ enterobactin does. ${ }^{20}$ PVDI and $\mathrm{PVD}_{\mathrm{G} 173}$ feature shorter exchange kinetics, which complicate the study of the stereospecificity of the transport chain as a whole; however, we do know that only $\Lambda$-type stuctures have, to date, been found in crystal structures of ferripyoverdine/FpvA complexes. ${ }^{19}$ Even so, for most siderophores, the point along the long and complex iron incorporation process at which a prospective stereospecificity is imposed is not clear. Interestingly, our calculations suggest that the recognition of siderophores by FpvA is agnostic to chirality: both stereoisomers of PVDI are equally favorably bound, while for $\mathrm{PVD}_{\mathrm{G} 173}$ the very clear preference for $\Lambda$ conformations in solution is completely neutralized following FpvA binding. This is consistent with our previous work ${ }^{17}$ which, comparing the binding free energies of FpvA to PVDI and $\mathrm{PVD}_{\mathrm{G} 173}$ in their $\Lambda$ states, concluded that the interactions of pyoverdines with water was more discriminating than their interactions with FpvA: this apparently applies not only to distinct pyoverdines, but also to different stereoisomers of the same siderophore. Keeping the previously mentioned caveats regarding exchange kinetics in mind, it also matches experimental findings that the TonB-dependent receptors FepA and FepB both bind enterobactin and enantio-enterobactin ${ }^{41}$, and that the enantiospecic step of the iron incorporation process is acted out later by the enterobactin hydrolase Fes. ${ }^{42}$ The ability to transport multiple chiralities of each siderophore regardless of whether their payload can be utilized in fine is a powerful means to sequester the siderophores of other bacterial strains under conditions of competitive growth.

Interestingly, the specificity of FpvA for pyoverdine strains seems to have been transferred away from the $\Lambda$ and $\Delta$ isomers to the 
intermediate state: the stability difference of solvated PVDI and $\mathrm{PVD}_{\mathrm{G} 173}$ intermediates is amplified by FpvA, resulting in tremendously divergent rate of exchange between chiralities. According to the Eyring-Polanyi theory, ${ }^{43}$ the corresponding isomerization timescales are $10 \mu \mathrm{s}$ for $\mathrm{PVD}_{\mathrm{G} 173}$ and $10^{6} \mathrm{~s}$ for PVDI (assuming first-order kinetics, respective activation free energies of 26 and $12 \mathrm{kcal} \mathrm{mol}^{-1}$ and a temperature of $\left.298 \mathrm{~K}\right) . \mathrm{PVD}_{\mathrm{G} 173}$ isomerization is hence much shorter than the typical pyoverdine transport time (in the range of minutes), ${ }^{44}$ meaning that chiral-specific steps further down along the transport chain (if any) are likely to be thermodynamic in nature. Facilitating the conversion from the ultra-dominant $\Lambda$ configuration to $\Delta$ could also be a way of fine-tuning the affinity of $P$. aeruginosa for this non-native pyoverdine, coralling its conformational preferences toward a common recognition framework that simultaneously matches several siderophores. Conversely, with conversion timescales orders of magnitude slower than transport timescales, the chirality of PVDI appears kinetically locked by FpvA, which could represent the onset of two separate pathways for the transport (or not) of the bacterium's native pyoverdine.

The varying accessibility of the metal ion in FpvA-bound pyoverdines is another important corollary of our work. Schons and coworkers ${ }^{16}$ have postulated that the pyoverdine peptide chain plays a more important role during the uptake of iron than in the recognition of the siderophore by FpvA. Indeed, in our simulations, the peptide chains of the $\Delta$ siderophores exhibit different folds from their $\Lambda$ counterparts and shield different zones around the metal ion. In $\Delta$ PVDI, $\mathrm{Fe}^{3+}$ is shielded from the outside medium by the bulk of the peptide chain and more easily accessed from the plug domain of the transporter, while in the $\Lambda$ siderophore the ion is more encumbered on the periplasmic than on the extracellular side. For $\mathrm{PVD}_{\mathrm{G} 173}$, the opposite is true: the metal is more accessible from the periplasmic side in the $\Lambda$ than in the $\Delta$ state. Could the folding of the chain inside the binding site act as a conformational switch, engaging or disengaging the ability to transport the siderophore by selectively shielding the presence of the metal ion to crucial recognition elements (for instance F795 and Y796, frequently found within $5 \AA$ of the ion)? If this is the case, and assuming that it explains the experimentally observed overall specificity of the iron transport chain for $\Lambda$ PVDI, does a similar specificity for $\mathrm{PVD}_{\mathrm{G} 173}$ exist? Such a preference would justify the stabilization of $\Delta \mathrm{PVD}_{\mathrm{G} 173}$ and the facilitated conversion from $\Lambda$ $\mathrm{PVD}_{\mathrm{G} 173}$ operated by FpvA, to help populate the $\Delta$ state which is almost nonexistent in solution. An example of such a behavior is known to occur in Bacillus subtilis, where the FeuA transporter only recognizes the less stable $\Delta$ state of siderophore bacillibactin but is able to convert the native $\Lambda$ state upon binding. ${ }^{45}$

Pyoverdines are often dubbed 'stealth sideophores', for their ability to evade the action of immune neutrophil cells that combat microbial infection by immobilizing metal scavengers. ${ }^{46}$ This is mostly due to the inability of the neutrophil-gelatinase-associated lipocalin protein (NGAL) to recognize and bind pyoverdines, ${ }^{47}$ which is attributed to electrostatic interactions within the NGAL binding site. ${ }^{48}$ Possible steric effects aside, the transition states between $\Lambda$ and $\Delta$ show similarities with bidentate catecholate ligands, whose affinity for NGAL is much degraded compared to tris- cathecolate ligands; the facilitated $\Lambda-\Delta$ isomerism could hence be one of the reasons behind the fact that pyoverdines are stealthy siderophores.

Finally, the siderophore-specific allosteric communication between the metal ion binding site and the FpvA lid loops could be used to couple the uptake of pyoverdines to other regulatory networks taking place in the outside medium. For instance, the ubiquitous Pseusomonas quinolone signal (PQS) which underpins cell-to-cell communication ${ }^{49}$ is also involved in iron metabolism: it can entrap $\mathrm{Fe}^{3+}$ near the outer membrane, facilitating its acquisition by siderophores, ${ }^{50}$ but also controls the enrichment of outer membrane vesicles in siderophores and TonB receptors... ${ }^{51}$ Despite its complexity, the siderophore-dependent motion of the FpvA loops certainly warrants further exploration.

\section{References}

1 L. M. Weiner, A. K. Webb, B. Limbago, M. A. Dudeck, J. Patel, A. J. Kallen, J. R. Edwards and D. M. Sievert, Infect. Control Hosp. Epidemiol., 2016, 37, 1288-1301.

2 M. D. Zilberberg and A. F. Shorr, J. Hosp. Med., 2013, 8, 559563.

3 P. D. Croughs, B. Li, J. A. A. Hoogkamp-Korstanje, E. Stobberingh and A. R. S. Group, Eur. J. Clin. Microbiol. Infect. Dis., 2013, 32, 283-288.

4 Global priority list of antibiotic-resistant bacteria to guide research, discovery, and development of new antibiotics, World health organization technical report, 2017.

5 P. Chandrangsu, C. Rensing and J. D. Helmann, Nat. Rev. Microbiol., 2017, 15, 338-350.

6 C. Cézard, N. Farvacques and P. Sonnet, Curr. Med. Chem., 2015, 22, 165-186.

7 R. Saha, N. Saha, R. S. Donofrio and L. L. Bestervelt, J. Basic Microbiol., 2013, 53, 303-317.

8 A. Braud, F. Hoegy, K. Jezequel, T. Lebeau and I. J. Schalk, Environ. Microbiol., 2009, 11, 1079-1091.

9 R. García-Contreras, B. Pérez-Eretza, E. Lira-Silva, R. JassoChávez, R. Coria-Jiménez, A. Rangel-Vega, T. Maeda and T. K. Wood, Pathog. Dis., 2014, 70, 95-98.

10 S. L. Hartney, S. Mazurier, M. K. Girard, S. Mehnaz, E. W. Davis, H. Gross, P. Lemanceau and J. E. Loper, J. Bacteriol., 2013, 195, 765-776.

11 J. Greenwald, M. Nader, H. Celia, C. Gruffaz, V. Geoffroy, J.M. M. Meyer, I. J. Schalk and F. Pattus, Mol. Microbiol., 2009, 72, 1246-1259.

12 A. S. Griffin, S. A. West and A. Buckling, Nature, 2004, 430, 1024-1027.

13 C. C. C. R. de Carvalho and P. Fernandes, Front. Microbiol., 2014, 5, 290. 
14 L. Moynié, A. Luscher, D. Rolo, D. Pletzer, A. Tortajada, H. Weingart, Y. Braun, M. G. P. Page, J. H. Naismith and T. Köhler, Antimicrob. Agents Chemother., 2017, 61, e02531-16.

15 G. S. Moeck and J. W. Coulton, Mol. Microbiol., 1998, 28, 675681.

16 V. Schons, R. A. Atkinson, C. Dugave, R. Graff, G. L. A. Mislin, L. Rochet, C. Hennard, B. Kieffer, M. A. Abdallah and I. J. Schalk, Biochemistry, 2005, 44, 14069-14079.

17 B. Bouvier, C. Cézard and P. Sonnet, Phys. Chem. Chem. Phys., 2015, 17, 18022-18034.

18 D.-L. L. Tzou, E. Wasielewski, M. A. Abdallah, B. Kieffer and R. A. Atkinson, Biopolymers, 2005, 79, 139-149.

19 E. Wasielewski, D.-L. L. Tzou, B. Dillmann, J. Czaplicki, M. A. Abdallah, R. A. Atkinson and B. Kieffer, Biochemistry, 2008, 47, 3397-3406.

20 B. Kersting, J. R. Telford, M. Meyer and K. N. Raymond, J. Am. Chem. Soc., 1996, 118, 5712-5721.

21 R. A. Atkinson, A. L. Salah El Din, B. Kieffer, J. F. Lefèvre and M. A. Abdallah, Biochemistry, 1998, 37, 15965-15973.

22 T. S. Piper, J. Am. Chem. Soc., 1961, 83, 3908-3909.

23 Y. Liu, Y. Liu and M. G. Drew, Coord. Chem. Rev., 2014, 260, 37-64.

24 A. D. Becke, Phys. Rev. A, 1988, 38, 3098-3100.

25 A. D. Becke, J. Chem. Phys, 1993, 98, 5648-5652.

26 C. Lee, W. Yang and R. G. Parr, Phys. Rev. B, 1988, 37, 785-789.

27 Y. Zhao and D. G. Truhlar, Acc. Chem. Res., 2008, 41, 157-167.

28 Y. Zhao and D. G. Truhlar, Theor. Chem. Acc., 2008, 120, 215241.

29 K. P. Kepp, Coord. Chem. Rev., 2013, 257, 196-209.

30 H. Hirao, N. Thellamurege and X. Zhang, Front. Chem., 2014, 2,14 .

31 V. Hornak, R. Abel, A. Okur, B. Strockbine, A. Roitberg and C. Simmerling, Proteins, 2006, 65, 712-725.

32 K. N. Kirschner, R. D. Lins, A. Maass and T. A. Soares, J. Chem. Theory Comput., 2012, 8, 4719-4731.

33 J. P. M. Jämbeck and A. P. Lyubartsev, J. Chem. Theory Comput., 2012, 8, 2938-2948.

34 W. L. Jorgensen, J. Chandrasekhar, J. D. Madura, R. W. Impey and M. L. Klein, J. Chem. Phys., 1983, 79, 926-935.
35 I. S. Joung and T. E. Cheatham, J. Phys. Chem. B, 2008, 112, 9020-9041.

36 D. H. de Jong, G. Singh, W. F. D. Bennett, C. Arnarez, T. A. Wassenaar, L. V. Schafer, X. Periole, D. P. Tieleman and S. J. Marrink, J. Chem. Theory Comput., 2012, 9, 687-697.

37 S. J. Marrink, H. J. Risselada, S. Yefimov, D. P. Tieleman and A. H. de Vries, J. Phys. Chem. B, 2007, 111, 7812-7824.

38 B. Van Oosten and T. A. Harroun, J. Mol. Graph. Model., 2016, 63, 125-133.

39 A. Barducci, G. Bussi and M. Parrinello, Phys. Rev. Lett., 2008, 100, 020603.

40 M. Shirley and I. L. Lamont, J. Bacteriol., 2009, 191, 56345640.

41 K. N. Raymond, B. E. Allred and A. K. Sia, Acc. Chem. Res., 2015, 48, 2496-2505.

42 R. J. Abergel, A. M. Zawadzka, T. M. Hoette and K. N. Raymond, J. Am. Chem. Soc., 2009, 131, 12682-12692.

43 D. J. Wales and P. Salamon, Proc. Natl. Acad. Sci. U S A, 2014, 111, 617-622.

44 I. J. Schalk, in New insights on iron acquisition mechanisms in pathogenic Pseudomonas, Springer, 2006, vol. 4, pp. 1-34.

45 F. Peuckert, M. Miethke, A. G. Albrecht, L.-O. O. Essen and M. A. Marahiel, Angew. Chem. Int. Ed. Engl., 2009, 48, 79247927.

46 P. Saha, B. S. Yeoh, R. A. Olvera, X. Xiao, V. Singh, D. Awasthi, B. C. Subramanian, Q. Chen, M. Dikshit, Y. Wang, C. A. Parent and M. Vijay-Kumar, J. Immunol., 2017, 198, 4293-4303.

47 M. E. Peek, A. Bhatnagar, N. A. McCarty and S. M. Zughaier, Interdiscip. Perspect. Infect. Dis., 2012, 2012, 843509.

48 C. Gómez-Casado, F. Roth-Walter, E. Jensen-Jarolim, A. DíazPerales and L. F. Pacios, J. Mol. Graph. Model., 2013, 45, 111121.

49 J. Lee and L. Zhang, Protein Cell, 2015, 6, 26-41.

50 S. P. Diggle, S. Matthijs, V. J. Wright, M. P. Fletcher, S. R. Chhabra, I. L. Lamont, X. Kong, R. C. Hider, P. Cornelis, M. Cámara and P. Williams, Chem. Biol., 2007, 14, 87-96.

51 N. Couto, S. R. Schooling, J. R. Dutcher and J. Barber, J Proteome Res, 2015, 14, 4207-22. 